\title{
Ulnar hemimelia
}

INSERM

\section{Source}

INSERM. (1999). Orphanet: an online rare disease and orphan drug data base. Ulnar hemimelia. ORPHA:93320

Ulnar hemimelia is a congenital ulnar deficiency of the forearm characterized by complete or partial absence of the ulna bone. 